\title{
Impact of COVID-19 on 'Living Well' with Mild-to-Moderate Dementia in the Community: Findings from the IDEAL Cohort
}

\author{
Linda Clare ${ }^{\mathrm{a}, \mathrm{b}, *}$, Anthony Martyr ${ }^{\mathrm{a}}$, Laura D. Gamble ${ }^{\mathrm{c}}$, Claire Pentecost ${ }^{\mathrm{a}}$, Rachel Collins ${ }^{\mathrm{a}}$, \\ Eleanor Dawson ${ }^{\mathrm{a}}$, Anna Hunt ${ }^{\mathrm{a}}$, Sophie Parker ${ }^{\mathrm{a}}$, Louise Allan ${ }^{\mathrm{a}}$, Alistair Burns ${ }^{\mathrm{d}}$, Alexandra Hillman ${ }^{\mathrm{e}}$, \\ Rachael Litherland $^{\mathrm{f}}$, Catherine Quinnn ${ }^{\mathrm{g}, \mathrm{h}}$, Fiona E. Matthews ${ }^{\mathrm{c}}$ and Christina Victor ${ }^{\mathrm{i}}$ \\ ${ }^{a}$ College of Medicine and Health, University of Exeter, Exeter, UK \\ ${ }^{\mathrm{b}}$ NIHR Applied Research Collaboration South-West Peninsula, Exeter, UK \\ ${ }^{\mathrm{c}}$ Population Health Sciences Institute, Newcastle University, Newcastle upon Tyne, UK \\ ${ }^{\mathrm{d}}$ Institute of Brain, Behaviour and Mental Health, University of Manchester, Manchester, UK \\ ${ }^{\mathrm{e}}$ Department of Public Health Policy and Social Sciences, Swansea University, Swansea, Wales, UK \\ ${ }^{\mathrm{f}}$ Innovations in Dementia CIC, Exeter, UK \\ ${ }^{\mathrm{g}}$ Centre for Applied Dementia Studies, Bradford University, Bradford, UK \\ ${ }^{\mathrm{h}}$ Wolfson Centre for Applied Health Research, Bradford, UK \\ ${ }^{\mathrm{i} C o l l e g e}$ of Health, Medicine and Life Sciences, Brunel University London, Uxbridge, UK
}

Handling Associate Editor: Andrea Tales

Accepted 27 October 2021

Pre-press 12 November 2021

\begin{abstract}
.
Background: Negative impacts of the COVID-19 pandemic on people with dementia have been widely-documented, but most studies have relied on carer reports and few have compared responses to information collected before the pandemic.

Objective: We aimed to explore the impact of the pandemic on community-dwelling individuals with mild-to-moderate dementia and compare responses with pre-pandemic data.

Methods: During the second wave of the pandemic, we conducted structured telephone interviews with 173 people with dementia and 242 carers acting as informants, all of whom had previously participated in the IDEAL cohort. Where possible, we benchmarked responses against pre-pandemic data.

Results: Significant perceived negative impacts were identified in cognitive and functional skills and ability to engage in self-care and manage everyday activities, along with increased levels of loneliness and discontinuity in sense of self and a decline in perceived capability to 'live well'. Compared to pre-pandemic data, there were lower levels of pain, depression, and anxiety, higher levels of optimism, and better satisfaction with family support. There was little impact on physical health, mood, social connections and relationships, or perceptions of neighborhood characteristics.

Conclusion: Efforts to mitigate negative impacts of pandemic-related restrictions and restore quality of life could focus on reablement to address the effects on participation in everyday activities, creating opportunities for social contact to reduce
\end{abstract}

\footnotetext{
${ }^{*}$ Correspondence to: Professor Linda Clare, College of Medicine and Health, University of Exeter, Exeter, UK. E-mail: 1.clare@ exeter.ac.uk; ORCIDs: 0000-0003-3989-5318.
} 
loneliness, and personalized planning to reconnect people with their pre-COVID selves. Such efforts may build on the resilience demonstrated by people with dementia and carers in coping with the pandemic.

Keywords: Alzheimer's disease, quality of life, services, well-being

\section{INTRODUCTION}

Multiple factors are associated with the way in which people living with dementia perceive their own quality of life [1] and ability to 'live well' [2] with the condition. 'Living well' refers to the 'best achievable state of health that encompasses all dimensions of physical, mental and social well-being' [2]. It is reflected in perceived quality of life, well-being, and satisfaction with life $[3,4]$. Grouping these multiple factors into life domains demonstrates two important points [3]. First, the life domains of psychological characteristics and health, social capitals, assets, and resources, social circumstances, physical fitness and health, and managing everyday life with dementia are all independently associated with perceived ability to 'live well' [4]. Second, when modelled together the psychological domain dominates. This suggests that experiences in multiple life domains are linked to perceived ability to 'live well' with the condition through their impact on psychological health, with varying combinations of factors explaining differences in individual outcomes [3].

These findings from the British IDEAL (Improving the experience of Dementia and Enhancing Active Life) cohort study offer an evidence-based framework for exploring the effects of the COVID-19 pandemic on people living with dementia in the community [4,5]. The emergence of the pandemic in 2020 and resulting periods of lockdown, interspersed with periods of ongoing social restriction, in the UK necessitated rapid changes to individual lifestyles, social engagement, and health and social care delivery. These changes could potentially affect several life domains associated with 'living well' for people with dementia in the community. Physical distancing and self-isolation could have an impact on social, mental, and physical health, and relationships. IDEAL data collected prior to the pandemic constitutes a unique benchmark against which to assess the extent of these effects among cohort participants.

Findings from a number of telephone or online surveys, mainly of informal carers, describe the perceived impact of the situation on people with dementia during the first wave of the pandemic.
Carers reported declines in the cognitive, communication, and functional abilities of people with dementia in several surveys [6-9]. Where asked, people with dementia themselves were concerned about declining skills and abilities [10-14]. Increases in neuropsychiatric symptoms, behavior problems, depression, anxiety, and loneliness were also reported by carers [11, 15-23], although some studies found only limited impacts in these areas [24, 25]. For example, Thyrian et al. [24] observed rates of depression, anxiety, and loneliness that were comparable to or even lower than age-equivalent norms for the general population of older people recorded before the pandemic. This highlights the importance of benchmarking data against pre-pandemic information or relevant population norms. Reduced availability of health care and support services had an impact during the early stages of the pandemic [26] leading to feelings of abandonment for some [12], with those living alone particularly affected [27]. However, there was little evidence for an immediate worsening of physical health [28].

It was important to gather evidence promptly in the exceptional situation created by the early stages of the pandemic in order to highlight the particular challenges faced by people living with dementia. However, the resulting set of evidence has three key limitations. First, few studies directly questioned people with dementia, relying instead on informant responses made by carers. Where people with dementia were included, their responses were often amalgamated with informant responses from carers. The perspectives of people with dementia and their carers can differ considerably and should not be used interchangeably when evaluating aspects of well-being [29]. Furthermore, in some instances carers may underestimate the abilities of the person with dementia or overestimate difficulties, while the responses of the person with dementia are more accurate relative to objective measures [30]. The reports carers provide when acting as informants are known to be affected by stress [31,32], and there is plenty of evidence to indicate that carers experienced elevated levels of stress during the pandemic [18, 33-38]. Second, only a handful of studies were able to offer 
any comparison with pre-pandemic measures and typically these reported on people participating in intervention trials, which tend to have highly selected samples, or those who had recently attended a memory clinic for assessment. Only one study [24] related responses to pre-pandemic population norms. Third, many studies focused on clinical symptoms with little attempt to gather data about the impact of the pandemic on quality of life or capability to 'live well' with dementia.

The need for restrictive measures to be in place over a lengthy period as the COVID-19 pandemic progressed to a second wave in Britain allows for an evaluation of impact over a longer timescale, taking into account the potential for adjustment after the initial stages. In the study reported here, we explored the impact of the pandemic on people with dementia who were part of the existing, well-documented IDEAL cohort, through interviews undertaken during the second wave of the pandemic. We aimed to address key limitations in the available evidence by: gathering and reporting information from both people with dementia and carers; comparing responses with prepandemic data; and evaluating a wide range of factors including effects on quality of life. Based on the available evidence, we anticipated that both people with dementia and carers acting as informants would describe a considerable degree of decline in cognitive and functional skills, and that benchmarking against data from pre-COVID assessments would demonstrate an impact on psychological health, ability to manage everyday activities, social engagement, and perceptions of capability to 'live well' with dementia.

\section{METHOD}

\section{Design}

We report a mixed-methods cross-sectional observational study embedded in, and forming a discrete component of, the ongoing longitudinal IDEAL cohort study. The present study, known as INCLUDE (Identifying and mitigating the individual and dyadic impact of COVID-19 and life under physical distancing on people with dementia and carers), focused on the experiences of people with dementia and carers from the IDEAL cohort during the pandemic. Participants living in England and Wales were interviewed between September 21, 2020 and April 30, 2021 using remote data collection methods.

Significant restrictions on social contact, mobility, retail, and services were in force throughout this time with long periods of full local or national lockdown, while the UK vaccination program for older and clinically vulnerable individuals began in December 2020.

All participants completed a structured telephone or online interview designed specifically for this study, which yielded both quantitative data and responses to open-ended questions; separate versions were prepared for people with dementia and for carers. A sub-set of participants additionally completed a semi-structured interview yielding qualitative data. Informed consent was documented for all participants.

In this paper we report quantitative data about the experiences of participants with dementia from the structured interviews, elicited through self-report and through informant reports given by carers. Where feasible, we drew comparisons with information collected from the IDEAL cohort prior to the pandemic. We also included for comparison purposes a small number of questions used in the English Longitudinal Study of Ageing (ELSA) COVID-19 Sub-Study Wave 2, for which data were collected in November and December 2020; these data are available for download from the UK Data Service [39].

The following section provides background information about the IDEAL cohort study. IDEAL recruited a cohort of people with mild-to-moderate dementia in Great Britain (i.e., England, Scotland, and Wales) between 2014 and 2016 (Time 1, T1) who are being followed up at regular intervals; in many cases, a family member or close friend (here termed 'carer') participates alongside the person with dementia. Details of the study can be found in the published protocols $[4,5]$. At baseline, the cohort comprised 1,537 community-dwelling individuals with dementia along with 1,277 carers. Inclusion criteria for people with dementia were a previouslyestablished clinical diagnosis of dementia and a current Mini-Mental State Examination (MMSE) [40] score of 15 or above (indicative of mild-tomoderate dementia) recorded as part of the study recruitment process. People with dementia and carers provided information during home visits by National Health Service (NHS) clinical research network staff. Both the distribution of dementia diagnoses, with Alzheimer's disease accounting for just over half of all diagnoses, and the small proportion of individuals from minority ethnic groups in the sample were consistent with available estimates of population values [41, 42]. From the original cohort, 1,183 people with dementia and 988 carers were interviewed again 
12 months later (2015-2017; T2) and 851 people with dementia and 759 carers after a further 12 months (2016-2018; T3). The fourth wave of data collection (2018-2020; T4), intended to follow 24 months after T3, was disrupted by the COVID-19 pandemic and not all participants were seen; at T4 data were collected from 253 people with dementia and 306 carers. The fifth wave of data collection (T5, planned to take place 12 months after T4, in 2019-2021) had to be discontinued at an early stage because home visits were no longer feasible. All IDEAL participants seen at $\mathrm{T} 4$, and those seen at $\mathrm{T} 3$ who had not yet been approached for T4, were invited to take part in INCLUDE.

Alongside T4, prior to the start of the pandemic, an additional 204 people with dementia were recruited to enrich the original IDEAL cohort, together with 183 carers (the 'enrichment group'). Recruitment of people with dementia to the enrichment cohort was restricted to those with frontotemporal and Parkinsonian dementias, those with young-onset dementia of any type $(<65$ years), and the very old $(90+$ years $)$ with dementia of any type; inclusion criteria were otherwise the same as for the original cohort. The intention when recruiting the enrichment group was to combine data collected at T4, T5, and T6 with data gathered from participants in the same sub-groups in the original cohort at $\mathrm{T} 1, \mathrm{~T} 2$, and $\mathrm{T} 3$, where numbers were small, for statistical analysis purposes. All enrichment group participants were invited to take part in INCLUDE.

INCLUDE was approved by Wales Research Ethics Committee 5 as an amendment to IDEAL-2 for England and Wales (18/WS/0111 AM12). IDEAL was approved by Wales Research Ethics Committee 5 (reference 13/WA/0405) and IDEAL-2 by Wales Research Ethics Committee 5 (reference 18/WS/ 0111) and Scotland A Research Ethics Committee (reference 18/SS/0037). IDEAL and IDEAL-2 are registered with the UK Clinical Research Network (UKCRN), numbers 16593 and 37955, respectively.

\section{Participants}

People living with dementia and their carers living in England or Wales who had previously participated in the IDEAL cohort study were eligible for INCLUDE if they had either taken part at the most recent assessment point or indicated willingness to be contacted again at the next assessment point. People with dementia could take part regardless of whether they had a participating carer, and carers could take part even if the person with dementia whom they supported was not taking part. The only exclusion criterion was a lack of capacity to give informed consent on the part of the person with dementia. For the purposes of the analyses presented here, which focus on people with dementia living in the community, data from carers reporting on a person with dementia who had moved into residential care since joining the cohort were excluded. Where people could not be contacted by telephone or email, an invitation letter was sent to the last known address.

\section{Measures}

The structured interview was designed specifically for the study in order to gather as much relevant information as possible across the life domains covered in IDEAL while limiting the burden on participants. Versions were prepared for people with dementia and carers. Carers were asked to both provide informant reports and describe their own experiences; the latter will be reported separately. The interviews were programmed in Qualtrics to facilitate data capture and management. They included brief measures and items from validated scales used in the IDEAL study, as well as bespoke questions focusing on experiences during the pandemic; some additional open-ended questions offered opportunities to expand on specific responses. To enable some comparison of experiences during the pandemic against data from a wider sample of the older population, we also included 7 questions from the English Longitudinal Study of Ageing (ELSA) COVID-19 Sub-Study Wave 2. All questions reported in this paper are listed in full in Supplementary Tables 3-6, with references to the sources from which they were taken provided where applicable. The interviews began with questions about health and healthcare during the pandemic, and subsequent sections covered perceptions of social connection and relationships, psychological health, ability to manage everyday life during this period, and overall perceptions of capability to 'live well'. To assess current cognitive function, we used the 5-minute Montreal Cognitive Assessment (MoCA) [43], which was designed to be suitable for telephone administration. In IDEAL, the MMSE was used on entry to the study and at each follow-up assessment, so for comparison purposes we converted MoCA total scores to their MMSE equivalents [44]. Demographic information was available from previously-collected data. 


\section{Procedure}

Trained interviewers who were graduate or masters level psychologists contacted potential participants to discuss the study by telephone, email, or video call according to individual preferences. Where people could not be contacted by telephone or email, an invitation letter was sent to the last known address containing a reply slip and stamped addressed envelope as well as details of how to contact the team by telephone or email. In initial conversations with participants, interviewers provided information about the study and answered questions to ensure that participants could make an informed decision about whether or not to participate. Informed consent was taken in a follow-up call unless there was any indication that the participant lacked capacity to decide about participation. The structured interviews for people with dementia who consented to participate were then conducted over the telephone or online via platforms such as Zoom or Microsoft Teams according to participant preference. Carers were given the option of self-completing the survey online; half took this option while the remainder were interviewed over the telephone or online. Interviews could be undertaken in one single meeting or several shorter meetings depending on participants' wishes. Protocols were in place to ensure appropriate responses should a participant become distressed during the interview and appropriate action should significant concerns arise about a participant's welfare.

\section{Data analysis}

Quantitative data were analyzed descriptively. Responses to questions or scales used previously in IDEAL were compared with data from the full cohort at T3, the most recent complete time-point, and non-parametric Chi-Square and Mann-Whitney U-tests were used to compare distributions across response categories or mean ratings to indicate where responses might differ pre- and post-COVID. The sample included both participants who had been in the cohort since the beginning and contributed data at T3 and participants who had joined the cohort as part of the enrichment group and who contributed data for the first time alongside the $\mathrm{T} 4$ round of data collection, and hence in relation to T3 data it comprised a mixture of paired and independent responses. Because of this partial overlap, we conducted sensitivity analyses using the 'PartiallyOverlapping' package in $\mathrm{R}$ which allows for a comparison of means using the partially overlapping $t$-test (for continuous and ordinal variables), and a comparison of proportions using the partially overlapping samples z-test (for dichotomous variables) [45-47]. In addition, differences for the paired sample only were examined using McNemar's tests and Friedman tests. These alternate methods have some limitations, with the PartiallyOverlapping package unable to handle categorical variables (these were treated as ordinal where appropriate), and McNemar's test unable to handle cells with low frequencies. Comparisons between INCLUDE and ELSA COVID-19 Wave 2 were conducted using Chi-Square and Mann-Whitney U-tests. ELSA COVID-19 Wave 2 cross-sectional weights for core members were applied $(n=5,558)$.

\section{RESULTS}

\section{Sample characteristics}

We identified 625 people with dementia and 584 carers from available records as potentially-eligible for INCLUDE. We were able to contact 516 people with dementia and 496 carers, which included 445 dyads. The final sample reported here comprised 173 people with dementia (116 from the original cohort and 57 from the enrichment group), and 242 carers (166 from the original cohort and 76 from the enrichment group. There were 126 dyads, 47 people with dementia who had no participating carer, and 1,16 carers who provided information about a person with dementia who did not contribute directly. A flowchart summarizing the recruitment process and reasons for withdrawal is provided in Supplementary Figure 1. Full details of the responses to all interview questions are provided for both the whole sample of people with dementia and carers and the subgroup of 126 dyads in Supplementary Tables 3-6. Sensitivity analyses (overlapping samples tests and paired samples tests) are reported in Supplementary Table 7. Here we focus on responses for the whole sample.

Characteristics of the participants with dementia and the carers contributing to the present analyses as informants are summarized in Table 1. Participants with dementia had a mean age of 74.33 years (range 50-98); 58.4\% were male, $94.8 \%$ were white British, and 21.4\% lived alone. The most common diagnosis was Alzheimer's disease (45.1\%) and the mean 5-minute MoCA cognitive test score was 18.74 (range 1-30; MMSE equivalent score 24.14 \pm 5.60 ). Overall, the MMSE equivalent score was higher for INCLUDE participants than the mean MMSE score 
Table 1

Characteristics of the participants with dementia and the carers who acted as informants

\begin{tabular}{|c|c|c|c|}
\hline & & $\begin{array}{c}\text { People with } \\
\text { dementia; } N(\%)\end{array}$ & Carers; $N(\%)$ \\
\hline \multirow[t]{2}{*}{$\overline{\mathrm{Sex}}$} & Male & $101(58.4)$ & $77(31.8)$ \\
\hline & Female & $72(41.6)$ & $165(68.2)$ \\
\hline \multirow{6}{*}{ Age } & $<65$ & $30(17.3)$ & $58(24.0)$ \\
\hline & $65-69$ & $25(14.5)$ & $45(18.6)$ \\
\hline & $70-74$ & $30(17.3)$ & $57(23.6)$ \\
\hline & $75-79$ & $32(18.5)$ & $31(12.8)$ \\
\hline & $80+$ & $56(32.4)$ & $51(21.1)$ \\
\hline & Mean (SD); range & $74.33(10.01) ; 50-98$ & $70.92(10.15) ; 32-93$ \\
\hline \multirow[t]{4}{*}{ Ethnicity } & White British & $164(94.8)$ & $226(93.4)$ \\
\hline & White Other & $4(2.3)$ & $7(2.9)$ \\
\hline & Other & $3(1.7)$ & $3(1.2)$ \\
\hline & Missing & $2(1.2)$ & $6(2.5)$ \\
\hline \multirow[t]{5}{*}{ Education } & No qualifications & $36(20.8)$ & $33(13.6)$ \\
\hline & school leaving certificate at age 16 & $32(18.5)$ & $55(22.7)$ \\
\hline & school leaving certificate at age 18 & $65(37.6)$ & $66(27.3)$ \\
\hline & University & $38(22.0)$ & $75(30.6)$ \\
\hline & Missing & $2(1.1)$ & $14(5.8)$ \\
\hline \multirow[t]{9}{*}{ Socioeconomic status $\wedge$} & I Professionals & $12(6.9)$ & $19(7.9)$ \\
\hline & II Managerial and technical & $67(38.7)$ & $95(39.3)$ \\
\hline & III-NM Skilled occupations - non-manual & $29(16.8)$ & $63(26.0)$ \\
\hline & III-M Skilled occupations - manual & $32(18.5)$ & $26(10.7)$ \\
\hline & IV Partly skilled & $20(11.6)$ & $13(5.4)$ \\
\hline & V Unskilled & $3(1.7)$ & $4(1.7)$ \\
\hline & Armed forces & $4(2.3)$ & $1(0.4)$ \\
\hline & N/A & $2(1.2)$ & $7(2.9)$ \\
\hline & Missing & $4(2.3)$ & $14(5.8)$ \\
\hline \multirow[t]{9}{*}{ Current marital status } & Single & $7(4.0)$ & $6(2.5)$ \\
\hline & Married; first & $88(50.9)$ & $156(64.5)$ \\
\hline & Remarried & $29(16.8)$ & $50(20.7)$ \\
\hline & A civil partnership & $2(1.2)$ & $2(0.8)$ \\
\hline & Legally separated & $2(1.2)$ & $2(0.8)$ \\
\hline & Divorced & $24(13.9)$ & $12(5.0)$ \\
\hline & Widowed & $15(8.7)$ & $1(0.4)$ \\
\hline & Cohabiting & $6(3.5)$ & $12(5.0)$ \\
\hline & Missing & - & $1(0.4)$ \\
\hline \multirow[t]{2}{*}{ Carer relationship } & Spouse/partner & & $210(86.8)$ \\
\hline & Other family/friend & & $32(13.2)$ \\
\hline \multirow{3}{*}{ Living situation } & Living alone & $37(21.4)$ & \\
\hline & Live with spouse/partner & $129(74.6)$ & \\
\hline & Live with other & $7(4.0)$ & \\
\hline \multirow[t]{7}{*}{ Diagnosis } & Alzheimer's disease (AD) & $78(45.1)$ & \\
\hline & Vascular dementia (VaD) & $17(9.8)$ & \\
\hline & Mixed $\mathrm{AD}$ and $\mathrm{VaD}$ & $26(15.0)$ & \\
\hline & Frontotemporal dementia (FTD) & $24(13.9)$ & \\
\hline & Parkinson's disease dementia (PDD) & $8(4.6)$ & \\
\hline & Dementia with Lewy bodies (DLB) & $14(8.1)$ & \\
\hline & Unspecified/Other & $6(3.5)$ & \\
\hline 5-minute MoCA & Mean (SD); range & $18.74(7.28) ; 1-30$ & \\
\hline Estimated MMSE equivalent to MoCA score & Mean (SD); range & $24.14(5.60) ; 9-30$ & \\
\hline
\end{tabular}

MoCA, Montreal Cognitive Assessment; MMSE, Mini-Mental State Examination. ${ }^{\wedge}$ Socioeconomic status is categorized in accordance with the standard method used in the UK, which is based on occupational classification [55].

at IDEAL T3 (IDEAL T3 $20.54 \pm 6.21, p<0.001$; Supplementary Table 7). However, considering only those INCLUDE participants who formed part of the original IDEAL cohort and were assessed at T3, the MMSE equivalent score was significantly lower in
INCLUDE than at IDEAL T3 $(23.99 \pm 5.80$ versus $25.18 \pm 3.67, p=0.019$; Supplementary Table 7). This is also demonstrated in Supplementary Figure 2, where the MMSE score from IDEAL T1-T3 is plotted for participants who were part of the original IDEAL 
Table 2

Experiences of health and healthcare during the pandemic, and comparison with benchmark data where available

\begin{tabular}{|c|c|c|c|c|c|}
\hline & \multicolumn{2}{|c|}{ INCLUDE } & \multirow{2}{*}{$\begin{array}{c}\text { Benchmark for } \\
\text { comparison where } \\
\text { applicable }\end{array}$} & \multicolumn{2}{|c|}{ Benchmark data } \\
\hline & $\begin{array}{c}\text { People with } \\
\text { dementia }\end{array}$ & $\begin{array}{c}\text { Carers } \\
\text { (informants) }\end{array}$ & & $\begin{array}{c}\text { People with } \\
\text { dementia }\end{array}$ & $\begin{array}{c}\text { Carers } \\
\text { (informants) }\end{array}$ \\
\hline \multicolumn{6}{|l|}{ Direct experience of COVID-19: } \\
\hline Had COVID-19 & $3.5 \%$ & $2.5 \%$ & Population* & $6.1 \%$ & $6.1 \%$ \\
\hline Treated in hospital for COVID-19 & $0.6 \%$ & $0.8 \%$ & ELSA+ & $0.5 \%$ & $0.5 \%$ \\
\hline Someone close to you had COVID-19 & $16.8 \%$ & $\mathrm{n} / \mathrm{a}$ & ELSA+ & $22.8 \%$ & \\
\hline \multicolumn{6}{|l|}{ Health during the pandemic: } \\
\hline Overall health poor or very poor & $11.0 \%$ & $12.0 \%$ & IDEAL T3 & $12.1 \%$ & \\
\hline Experienced moderate or extreme pain & $44.5 \%$ & $46.7 \%$ & IDEAL T3 & $37.8 \%$ & $55.3 \%$ \\
\hline Developed new health problems & $22.0 \%$ & $\mathrm{n} / \mathrm{a}$ & & & \\
\hline Avoided seeking help for health issues & $19.7 \%$ & $7.4 \%$ & & & \\
\hline Healthcare needs affected by pandemic & $48.0 \%$ & $37.6 \%$ & & & \\
\hline Healthcare services stopped due to pandemic & $13.9 \%$ & $15.3 \%$ & & & \\
\hline \multicolumn{6}{|l|}{ Contact with health professionals and services: } \\
\hline Seen GP in person in last 3 months & $23.7 \%$ & $19.0 \%$ & IDEAL T3 & & $58.5 \%$ \\
\hline Spoken to GP telephone in last 3 months & $37.6 \%$ & $\mathbf{5 0 . 0 \%}$ & IDEAL T3 & & $16.1 \%$ \\
\hline Named health professional available & $\mathrm{n} / \mathrm{a}$ & $62.0 \%$ & IDEAL T3 & & $35.2 \%$ \\
\hline Health professional available due to dementia & $\mathrm{n} / \mathrm{a}$ & $\mathbf{5 0 . 8 \%}$ & IDEAL T3 & & $22.9 \%$ \\
\hline Attended day center (pre COVID-related closure) & $12.1 \%$ & $15.7 \%$ & IDEAL T3 & & $19.2 \%$ \\
\hline Day center remains closed & $8.1 \%$ & $11.1 \%$ & IDEAL T3 & & \\
\hline
\end{tabular}

ELSA, English Longitudinal Study of Ageing; IDEAL, Improving the experience of Dementia and Enhancing Active Life. Bold type indicates a statistically significant difference. *Population estimate for people aged 50+in England as of 8 May 2021; source: https://coronavirus.data.gov.uk/details/download + English Longitudinal Study on Ageing COVID Sub-Study Wave 2 core members [39].

cohort and hence had MMSE scores at those timepoints, together with the MoCA-equivalent MMSE score from INCLUDE. This shows, at group level, a gradual decline in MMSE score over time with no indication of a steeper decline during the pandemic. Among the carers, $86.8 \%$ were spouses or partners of the person with dementia. Carers had a mean age of 70.92 years (range $32-93$ ), and $68.2 \%$ were female. Characteristics of participants in IDEAL T3 and the ELSA COVID-19 Sub-Study Wave 2, the data from which were used in benchmarking, are shown in Supplementary Tables 1 and 2 .

\section{Health and healthcare during the pandemic}

Details of responses to questions about health and healthcare are summarized in Table 2; full details can be found in Supplementary Table 3. According to carers, $5.9 \%$ of people with dementia had been infected with COVID-19 prior to the interview, a proportion which was in line with population data for over 50s in England as of May 8, 2021. Among the respondents with dementia, $3.5 \%$ reported having been infected with COVID-19 and $16.8 \%$ said someone close to them had been infected with the virus, which was lower than the $22.8 \%$ reported in the general older population in the ELSA COVID-19
Sub-Study Wave 2. Twenty-two percent of the participants with dementia said they had developed new health problems during the pandemic and a similar proportion $(19.7 \%)$ said they had avoided seeking help because of COVID-19, although only $7.4 \%$ of carers thought there had been an avoidance of helpseeking. Within dyads, more people with dementia than carers reported avoiding seeking help (15.1\% versus $10.3 \%$ ). Despite this, for the participants with dementia, self-ratings of health in the past 4 weeks did not differ significantly from equivalent ratings at IDEAL T3 (11\% versus $12.1 \%$ endorsing poor or very poor). While self-ratings of pain and mobility did not differ significantly from IDEAL T3, carer ratings indicated significantly fewer problems with pain ( $46.7 \%$ versus $55.3 \%$ ). This was supported in the partially overlapping samples test, but not when looking at paired samples only (Supplementary Table 7).

About half of the participants with dementia (48\%) thought their health care needs had been affected by the pandemic, for example through appointments being postponed or operations delayed, and within dyads perceptions were similar. Only $13.9 \%$ of people with dementia and $15.3 \%$ of carers reported that healthcare services people with dementia were already receiving had stopped due to the pandemic. Nearly one-quarter of the participants with dementia 
Table 3

Cognition, functioning, and managing everyday activities during the pandemic, and comparison with benchmark data where available

\begin{tabular}{|c|c|c|c|c|c|}
\hline & \multicolumn{2}{|c|}{ INCLUDE } & \multirow{2}{*}{$\begin{array}{c}\text { Benchmark for } \\
\text { comparison where } \\
\text { applicable }\end{array}$} & \multicolumn{2}{|c|}{ Benchmark data } \\
\hline & $\begin{array}{l}\text { People with } \\
\text { dementia }\end{array}$ & $\begin{array}{c}\text { Carers } \\
\text { (informants) }\end{array}$ & & $\begin{array}{c}\text { People with } \\
\text { dementia }\end{array}$ & $\begin{array}{c}\text { Carers } \\
\text { (informants) }\end{array}$ \\
\hline \multicolumn{6}{|l|}{ Cognitive and functional ability: } \\
\hline Memory poor or very poor & $30.7 \%$ & $\mathrm{n} / \mathrm{a}$ & IDEAL T3 & $23.4 \%$ & \\
\hline Difficulty with everyday activities & $39.3 \%$ & $69.1 \%$ & IDEAL T3 & $36.4 \%$ & $76.6 \%$ \\
\hline Difficulty with self-care & $28.3 \%$ & $54.6 \%$ & IDEAL T3 & $21.0 \%$ & $49.9 \%$ \\
\hline Problems with mobility & $44.5 \%$ & $60.8 \%$ & IDEAL T3 & $42.2 \%$ & $58.4 \%$ \\
\hline Perceived impact of pandemic on cognition: & $43.9 \%$ & $77.7 \%$ & & & \\
\hline Ability to remember everyday things declined & $45.1 \%$ & $73.1 \%$ & & & \\
\hline Ability to remember recent events declined & $49.1 \%$ & $70.2 \%$ & & & \\
\hline Ability to concentrate declined & $42.8 \%$ & $63.2 \%$ & & & \\
\hline Ability to say what you want to say declined & $31.8 \%$ & $49.2 \%$ & & & \\
\hline Ability to plan ahead declined & $30.6 \%$ & $55.0 \%$ & & & \\
\hline Ability to make decisions declined & $47.4 \%$ & $77.3 \%$ & & & \\
\hline Felt confused more often & $43.9 \%$ & $77.7 \%$ & & & \\
\hline \multicolumn{6}{|l|}{ Occupation: } \\
\hline Use internet & $68.8 \%$ & $38.0 \%$ & IDEAL T3 & $28.8 \%$ & $24.3 \%$ \\
\hline Fairly or very easy to keep oneself occupied & $81.5 \%$ & $65.3 \%$ & & & \\
\hline
\end{tabular}

Improving the experience of Dementia and Enhancing Active Life, IDEAL. Bold type indicates a statistically significant difference.

said they had seen a General Practitioner (GP) in person in the last 3 months, and over one-third said they had spoken to a GP on the telephone. Carer-reported contact with GPs reflected the shift to telephone consultations, with in person appointments significantly less frequent but telephone appointments significantly more frequent compared to IDEAL T3; these findings were supported in the sensitivity analyses shown in Supplementary Table 7. The overall level of contact was somewhat lower than at IDEAL T3 $(66.1 \%$ versus $77.5 \%)$. More carers said they had a named health professional whom they could contact at any time than was the case in IDEAL T3 $(62.0 \%$ versus $35.2 \%$ ), and the named health professional was more often in place due to the dementia diagnosis rather than another health condition than at IDEAL T3 (50.8\% versus $22.9 \%$ ). These findings were supported in the paired samples tests (Supplementary Table 7).

\section{Cognition, functioning, and managing everyday activities}

Responses to questions about cognition, functioning and managing everyday activities are summarized in Table 3; full details can be seen in Supplementary Tables 4 and 7. Considerable proportions of people with dementia had noticed a decline in their ability to remember everyday things (43.9\%), remember recent events $(45.1 \%)$, concentrate $(49.1 \%)$, say what they wanted to say (42.8\%), plan ahead (31.8\%), and make decisions (30.6\%), and increased confusion (47.4\%). Higher proportions of carers reported a worsening in ability to remember everyday things $(77.7 \%)$, remember recent events $(73.1 \%)$, concentrate $(70.2 \%)$, use language $(63.2 \%)$, plan ahead $(49.2 \%)$, and make decisions (55.0\%), and increased confusion (77.3\%), among people with dementia.

Self-ratings of day-to-day memory functioning were significantly different to those of the IDEAL T3 sample, with more endorsing 'poor' or 'very poor' (30.7\% versus $23.4 \%$ ) but also slightly more endorsing 'very good' or 'excellent' (13.3\% versus $8.6 \%)$. This finding was supported in the tests for paired samples only (Supplementary Table 7). The majority of people with dementia $(58.4 \%)$ perceived no problems in doing everyday activities, and about onethird (35.8\%) thought they had 'some problems'; this did not differ significantly from responses at IDEAL T3 (Supplementary Table 4 and Supplementary Figure 2). Carers perceived significantly more difficulties, with nearly one-quarter saying that the person with dementia was unable to carry out usual activities (23.6\%), which was supported in the sensitivity analysis (Supplementary Table 7). This is also demonstrated in Supplementary Figure 2, where the score on this question from IDEAL T1-T3 is plotted for participants who were part of the original IDEAL cohort, together with the equivalent score from INCLUDE. It shows carers reporting a gradual 
Table 4

Social capitals, assets, and resources during the pandemic, and comparison with benchmark data where applicable

\begin{tabular}{|c|c|c|c|c|c|}
\hline & \multicolumn{2}{|c|}{ INCLUDE } & \multirow{2}{*}{$\begin{array}{c}\text { Benchmark for } \\
\text { comparison where } \\
\text { applicable }\end{array}$} & \multicolumn{2}{|c|}{ Benchmark data } \\
\hline & $\begin{array}{l}\text { People with } \\
\text { dementia }\end{array}$ & $\begin{array}{c}\text { Carers } \\
\text { (informants) }\end{array}$ & & $\begin{array}{c}\text { People with } \\
\text { dementia }\end{array}$ & $\begin{array}{c}\text { Carers } \\
\text { (informants) } \\
\end{array}$ \\
\hline \multicolumn{6}{|l|}{ Practical concerns: } \\
\hline Worried about having enough food & $8.1 \%$ & $5.8 \%$ & $\mathrm{ELSA}^{+}$ & $8.1 \%$ & $8.1 \%$ \\
\hline Financially worse off & $9.8 \%$ & $\mathrm{n} / \mathrm{a}$ & $\mathrm{ELSA}^{+}$ & $18.9 \%$ & \\
\hline \multicolumn{6}{|l|}{ Family and friends: } \\
\hline Get on very or extremely well with carer (dyads) & $76.2 \%$ & $71.4 \%$ & IDEAL T3 & $68.5 \%$ & $65.6 \%$ \\
\hline Number of relatives in contact at least monthly & $5.6 \pm 4.2$ & $5.0 \pm 3.5$ & IDEAL T3 & $4.7 \pm 3.7$ & $4.8 \pm 3.6$ \\
\hline Very/slightly satisfied with support from family & $85.0 \%$ & $\mathrm{n} / \mathrm{a}$ & IDEAL T3 & $80.2 \%$ & \\
\hline Number of friends in contact at least monthly & $5.1 \pm 6.1$ & $3.6 \pm 4.5$ & IDEAL T3 & $5.2 \pm 7.3$ & $4.1 \pm 4.5$ \\
\hline Very/slightly satisfied with support from friends & $76.9 \%$ & $\mathrm{n} / \mathrm{a}$ & IDEAL T3 & $63.5 \%$ & \\
\hline \multicolumn{6}{|l|}{ Neighborhood: } \\
\hline People in neighborhood willing to help & $75.7 \%$ & $\mathrm{n} / \mathrm{a}$ & IDEAL T3 & $71.5 \%$ & \\
\hline People in neighborhood trustworthy & $74.6 \%$ & $\mathrm{n} / \mathrm{a}$ & IDEAL T3 & $69.2 \%$ & \\
\hline Satisfied with neighborhood & $90.7 \%$ & $\mathrm{n} / \mathrm{a}$ & IDEAL T3 & $84.7 \%$ & \\
\hline
\end{tabular}

ELSA, English Longitudinal Study of Ageing; IDEAL, Improving the experience of Dementia and Enhancing Active Life. Bold type indicates a statistically significant difference. ${ }^{+}$English Longitudinal Study on Ageing COVID Sub-Study Wave 2 core members [39].

increase in difficulty with everyday activities over time, but the increase in the number of people with dementia said to be unable to carry out these activities at the time of the INCLUDE assessment might reflect a somewhat steeper decline than would otherwise be expected, although it is not possible to establish this with any certainty. Over two-thirds of people with dementia thought they had no difficulties with self-care $(69.4 \%)$ and one-quarter $(24.3 \%)$ thought they had 'some' problems; this differed significantly from IDEAL T3 where only $16.3 \%$ perceived some difficulties. Carers perceived significantly more difficulties in self-care, with just under one-fifth (18.2\%) saying the person with dementia was unable to wash or dress independently, and this was supported in the sensitivity analysis.

Nearly three-quarters of participants with dementia $(81.5 \%)$ felt it had been very or fairly easy to keep themselves occupied during the pandemic, and two-thirds of carers $(65.3 \%)$ shared this view. Over two-thirds (68.8\%) of people with dementia said they used the internet, compared to only $28.8 \%$ at IDEAL T3; informant reports suggested that fewer people with dementia were using the Internet, although there was still an increase compared to IDEAL T3 $(38.0 \%$ versus $24.3 \%$ ). These findings were supported in the sensitivity analyses.

\section{Social capitals, assets, and resources}

Details of responses to questions about social capitals, assets, and resources are summarized in Table 4 and Supplementary Table 5. People with dementia tended to be more satisfied with the support they received from family compared to responses in IDEAL T3 (85.0\% versus $80.2 \%$ very or slightly satisfied), and the average number of family members in contact was slightly higher. Sensitivity analyses support the findings that more family members were in contact since IDEAL T3, but not the finding that people with dementia tend to be more satisfied with support from family (Supplementary Table 7). Generally, the relationship with the primary carer was perceived positively by those with a carer participating in the study, with over three-quarters (76.2\%) saying they got on 'very' or 'extremely' well, and carers also endorsed the perception of positive relationships $(71.4 \%)$; there were no significant differences to the ratings made at IDEAL T3. The number of friends in regular contact did not differ significantly from IDEAL T3 for people with dementia; carer ratings suggested a small but statistically significant reduction compared to IDEAL T3, also supported in the paired samples only test (Supplementary Table 7). Although a higher proportion expressed satisfaction with support from friends $(76.9 \%$ versus $63.5 \%)$ than at IDEAL T3, this did not differ significantly. Similarly, although higher proportions gave positive ratings of neighborhood trust ( $74.6 \%$ versus $69.2 \%$ ) and willingness to help (75.7\% versus $71.5 \%)$, and satisfaction with the neighborhood as a place to live $(90.7 \%$ versus $84.7 \%$ ), there were no significant differences to IDEAL T3. 
Table 5

Psychological well-being and quality of life during the pandemic, and comparison with benchmark data where applicable

\begin{tabular}{|c|c|c|c|c|c|}
\hline & \multicolumn{2}{|c|}{ INCLUDE } & \multirow{2}{*}{$\begin{array}{c}\text { Benchmark for } \\
\text { comparison where } \\
\text { applicable }\end{array}$} & \multicolumn{2}{|c|}{ Benchmark data } \\
\hline & $\begin{array}{l}\text { People with } \\
\text { dementia }\end{array}$ & $\begin{array}{c}\text { Carers } \\
\text { (informants) }\end{array}$ & & $\begin{array}{l}\text { People with } \\
\text { dementia }\end{array}$ & $\begin{array}{c}\text { Carers } \\
\text { (informants) }\end{array}$ \\
\hline \multicolumn{6}{|l|}{ Negative feelings: } \\
\hline Moderately or extremely anxious or depressed & $26.6 \%$ & $33.5 \%$ & IDEAL T3 & $31.2 \%$ & $47.6 \%$ \\
\hline Anxiety the previous day $(0-10$ scale $)$ & $3.0 \pm 3.1$ & $\mathrm{n} / \mathrm{a}$ & $\mathrm{ELSA}^{+}$ & $3.0 \pm 2.9$ & \\
\hline Not the same person I have always been & $29.5 \%$ & $\mathrm{n} / \mathrm{a}$ & IDEAL T3 & $20.3 \%$ & \\
\hline Feel lonely & $38.7 \%$ & $\mathrm{n} / \mathrm{a}$ & IDEAL T3 & $20.2 \%$ & \\
\hline \multicolumn{6}{|l|}{ Positive feelings: } \\
\hline Cheerful \& in good spirits $>50 \%$ of time last $2 \mathrm{wks}$ & $71.7 \%$ & $62.0 \%$ & IDEAL T3 & $77.5 \%$ & $61.4 \%$ \\
\hline Happiness the previous day $(0-10$ scale $)$ & $7.4 \pm 2.2$ & $\mathrm{n} / \mathrm{a}$ & $\mathrm{ELSA}^{+}$ & $7.2 \pm \mathbf{2 . 0}$ & \\
\hline Expect more good things to happen than bad & $71.1 \%$ & $\mathrm{n} / \mathrm{a}$ & IDEAL T3 & $64.5 \%$ & \\
\hline \multicolumn{6}{|l|}{ Coping with the pandemic: } \\
\hline Pandemic had positive aspects or benefits & $45.1 \%$ & $22.3 \%$ & & & \\
\hline Coped very or fairly well during the pandemic & $91.4 \%$ & $84.3 \%$ & & & \\
\hline \multicolumn{6}{|l|}{ Quality of life: } \\
\hline Satisfied with life $(0-10$ scale $)$ & $7.0 \pm 2.2$ & $\mathrm{n} / \mathrm{a}$ & IDEAL T3 & $7.7 \pm 2.1$ & \\
\hline Feel the things I do are worthwhile $(0-10)$ & $7.2 \pm 2.2$ & $\mathrm{n} / \mathrm{a}$ & IDEAL T3 & $7.8 \pm 2.0$ & \\
\hline Feel life as a whole is good or excellent & $68.8 \%$ & $50.8 \%$ & IDEAL T3 & $79.4 \%$ & $47.3 \%$ \\
\hline
\end{tabular}

ELSA, English Longitudinal Study of Ageing; IDEAL, Improving the experience of Dementia and Enhancing Active Life. Bold type indicates a statistically significant difference. ${ }^{+}$English Longitudinal Study on Ageing COVID Sub-Study Wave 2 core members [39].

With regard to practical concerns, the proportion of carers reporting worries about having enough food during the pandemic was slightly higher than for the ELSA sample (5.8\% versus $8.1 \%$ ) and there was a statistically-significant difference; the proportion of participants with dementia who said they had been worried about having enough food was the same as that in the ELSA sample (8.1\%). In relation to their financial situation, significantly fewer people thought they were worse off compared to before the pandemic than was the case in ELSA.

\section{Psychological well-being}

Responses to questions about psychological wellbeing are summarized in Table 5, and additional details are given in Supplementary Table 6A. Most people with dementia said they did not feel depressed or anxious. Rates of depression and anxiety were slightly lower than at IDEAL T3 $(26.6 \%$ versus $31.2 \%$ ) but did not differ significantly, and anxiety levels the previous day were in line with the wider population surveyed in ELSA. Carers perceived significantly lower levels of depression and anxiety than was the case at IDEAL T3 (33.5\% versus $47.6 \%)$ which was supported in the partially overlapping samples test (Supplementary Table 7). Nearly threequarters of people with dementia said that in the last 2 weeks they had felt cheerful and in good spirits more than half of the time, most of the time or all the time, and although the proportion was lower than at IDEAL T3 $(71.7 \%$ versus $77.5 \%$ ), this did not differ significantly. Carers had a slightly less positive view, but this did not differ significantly from IDEAL T3 $(62.0 \%$ versus $61.4 \%$ ). Ratings of happiness the previous day did not differ significantly from the ratings obtained in ELSA. People with dementia were significantly more optimistic than was the case in IDEAL T3, with $71.1 \%$ versus $64.5 \%$ expecting more good things to happen to them than bad, which was again supported in the partially overlapping test. Nearly half $(45.1 \%)$ of the participants with dementia, but only around one-fifth $(22.3 \%)$ of carers, thought there had been some positive aspects or benefits of the COVID-19 pandemic. Most people with dementia thought they had coped fairly or very well during the pandemic, with nearly half (48.6\%) endorsing 'very well' and only $6.9 \%$ saying they had not coped well; carers were somewhat less positive, with just over one-third (38.8\%) endorsing 'very well' and $14.9 \%$ saying the person with dementia had not coped well.

In contrast to the generally positive picture with regard to perceptions of mood and coping, people with dementia reported significantly higher rates of loneliness compared to IDEAL T3 (38.7\% versus $20.2 \%$ ), and significantly more disagreed with the statement that they were 'still the same person' they had always been ( $29.5 \%$ versus $20.3 \%$ ). Both of these findings were supported in sensitivity analyses (Supplementary Table 7). 


\section{Capability to 'live well' with dementia}

Responses to questions about capability to "live well' are summarized in Table 5 with additional details in Supplementary Table 6B. People with dementia reported significantly lower satisfaction with life, and less of a sense that the things they do are worthwhile compared to IDEAL T3. When asked how they feel about their life as a whole, fewer endorsed 'good' or 'excellent' than was the case in IDEAL T3 (68.8\% versus 79.4\%). Carers, although having a less positive view overall, were slightly more likely to endorse 'good' or 'excellent' than at IDEAL T3 (50.8\% versus $47.3 \%)$. All of these findings were supported in the sensitivity analyses (Supplementary Table 7).

\section{DISCUSSION}

Availability of the well-documented IDEAL cohort provided a unique opportunity to examine the perceived impact of the COVID-19 pandemic on people with dementia living in the community in Britain, with potential to compare responses with data collected before the pandemic. Mirroring the broad focus of the IDEAL programme, interviews undertaken during the second wave of the pandemic covered experiences in a range of life domains and perceived capability to 'live well' with dementia. Responses from the participants with dementia and from carers acting as informants suggested they perceived little negative impact on physical health, mood, social connections and relationships, or perceptions of neighborhood characteristics. Indeed, compared to benchmark data, there were indications of lower levels of pain, depression and anxiety, higher levels of optimism, and better satisfaction with family support. As expected, negative impacts were perceived in relation to cognitive and functional skills, and ability to engage in self-care and manage everyday activities, along with increased levels of loneliness, a higher proportion feeling 'not the same person' as before, and an overall decline in perceptions of capability to 'live well' with dementia.

There was no evidence in our sample of even a modest perceived negative impact on physical health. This reflected indications gathered in the early stages of the pandemic [27, 28], and was observed despite perceptions that healthcare needs were affected by the pandemic and reports of developing new health problems. The lack of reports of any negative impact was reassuring given early concerns that negative conse- quences for physical health might not be detectable immediately but might become evident over a longer time-period [48]. One factor that possibly mitigated against a negative impact on physical health in our sample was the availability of GP telephone consultations and an increase in the proportion having a named health professional as a key point of contact.

There was also no evidence of perceived negative impacts on social capitals, assets, and resources, despite reduced availability of support services and usual activities during lockdown periods and the potential for increased tension on caregiving relationships due to lack of opportunity for respite. Our findings were consistent with other indications that social support was generally satisfactory and that people found alternative activities to engage in where usual activities were not available [24]. Although carers acting as informants were more likely to indicate concern over availability of food during the pandemic than the general population of over 50s, perceptions of neighborhood characteristics were generally positive.

Evidence regarding psychological well-being was mixed. Importantly, we did see evidence of increased rates of feeling lonely relative to pre-COVID responses, consistent with reports of increased loneliness during the first wave of the pandemic $[11,15,17-$ 19, 23]. Other negative psychological impacts of the pandemic were suggested by the increased proportion of people with dementia feeling they were 'not the same person' as before; previous work with IDEAL cohort data identified this question as a sensitive indicator of poor psychological well-being [49]. However, reported levels of depression and anxiety did not differ significantly from benchmark except that carers were less likely to think that the person with dementia was depressed or anxious. Similarly, ratings of positive mood did not differ significantly from benchmark, except that people with dementia were significantly more optimistic. These findings appear to conflict with the numerous reports of increased levels of anxiety and depression in the first wave [11, 15, 17-19, 23], but may reflect changes in perspective as people adjusted to the pandemic, and reasons to feel optimistic once effective vaccines became available and vaccination programs were introduced by the UK NHS.

As expected, there were responses suggesting strong perceptions of decline in cognitive and functional ability from both people with dementia and informants, and compared to benchmark data there were increased levels of perceived memory problems 
and difficulties with self-care. This was consistent with earlier studies primarily based on carer report that highlighted declines in cognitive [7, 8, 17, 22], functional [6, 8, 17], and communication [9] ability during the pandemic. Responses of people with dementia in our study were consistent with early reports indicating that people with dementia themselves were concerned about increased decline in abilities during lockdown [11, 13, 14]. While some decline would be expected over time, perceptions of accelerated cognitive decline are supported by studies of memory clinic samples that identified steeper trajectories of decline in MMSE scores during the pandemic [50,51]. Perceived decline in cognitive and functional abilities over and above what would normally be expected could be attributable to the effects of pandemic-related restrictive measures, including more social isolation, reduction in usual activities and loss of routine as well as increased carer stress [51]. However, for those INCLUDE participants who contributed at IDEAL T1-T3, comparison with prepandemic data suggests that decline in cognitive ability was gradual over time, with no indication of a sudden steep drop during the pandemic; the situation is less clear with regard to carers' ratings of difficulty with everyday activities, where the increase in reports of people with dementia being unable to carry out these activities may possibly suggest a steeper decline than would otherwise be expected.

Also as expected, responses indicated significant perceived negative impacts of the pandemic on capability to 'live well' with dementia from the perspective of participants with dementia, although not from that of carers acting as informants. Given that multiple life domains, and individual factors within those domains, are associated with capability to 'live well' [3], these perceptions may be linked to perceived negative impacts of the pandemic in the domains of managing everyday life with dementia and psychological well-being. Having poorer functional ability, along with being lonely and feeling 'not the same person' have all been associated with poorer quality of life, satisfaction with life, and wellbeing in previous analyses of IDEAL cohort data [32, 49, 52].

The findings outlined above must be considered in the context of study limitations. While comparison with benchmark data is a useful indicator of pandemic-related effects, we needed to use the most recent complete dataset, rather than $\mathrm{T} 4$ which was interrupted by the start of the pandemic. IDEAL T3 data collection was completed 18 months prior to the outbreak of COVID-19, and hence other changes in the intervening time period may have influenced responses. For example, the higher proportion of carers saying the person with dementia had a named health professional as a main point of contact may reflect the emphasis on ensuring availability of a named health professional in updated practice guidance on management of dementia [53]. The differences observed are not necessarily caused by or attributable to the COVID-19 pandemic.

Additional recruitment to enrich the IDEAL cohort at T4 meant that the characteristics of the current sample, although similar in most respects, were different in some ways to the sample at IDEAL T3. The current sample had a higher proportion of people aged under 65 (17.3\% versus $7.8 \%)$ and higher proportions with frontotemporal dementia (13.9\% versus $3.7 \%)$, Parkinson's disease dementia ( $4.6 \%$ versus $2 \%)$, and dementia with Lewy bodies (8.1\% versus $3.2 \%$ ). Higher numbers of people with these rarer dementias where memory problems are less prominent in the early stages might account for the increased proportion reporting perceived 'good' or 'excellent' memory. A higher proportion of the enrichment group declined to participate in the current study than was the case among the original cohort members, and those participants from the original cohort who remain involved in the study after several years of participation may be a particularly resilient group with good physical health. The main reasons for attrition from IDEAL T1 to IDEAL T3 were increased health problems or death. It is possible therefore that our data underestimate the negative impact of the pandemic. Nevertheless, we did observe significant perceived negative effects in expected areas.

The significant results reported are intended to indicate variables which may show changes from pre-pandemic levels. The statistical tests used in the study assumed the current (INCLUDE) and IDEAL T3 samples were independent of each other, but they were partially overlapping and incorporated both paired and unpaired samples. We therefore conducted sensitivity analyses using the partially overlapping $t$ test or z-test, and paired samples tests on paired data only. The partially overlapping tests are not able to handle categorical variables but since the categorical variables we tested were ordered, they were treated as ordinal, and the $t$-test was conducted as recommended by Derrick et al. [47]. The tests for paired samples only also had limitations as the McNemar's test used to handle categorical variables fails when cells have zero or low numbers. When the partially overlapping 
samples tests or paired samples tests conducted for purposes of sensitivity analysis do not support the results of the main analyses, the main findings should be interpreted with caution, but in addition the limitations of these additional tests should be borne in mind. Finally, in designing the structured interviews we had to be mindful of the potential burden on participants and in order to cover the broad range of areas of interest and allow participants space to describe their experiences we opted to assess key constructs through single items rather than scales and had to take some difficult decisions about which topics to leave out.

Despite these limitations the study has a number of strengths. The sample is a sizeable one and conveys the perspectives of both people with dementia themselves and carers acting as informants, as well as allowing for benchmarking of responses. The data were collected over a seven-month period during the second wave of the pandemic and may present a more realistic picture than that obtained amid the heightened emotion and anxiety of the first few weeks of restrictions, as there had been time for people to adjust. Because the study is embedded in the IDEAL programme, it will be possible to follow up the participants in a further round of data collection. This is important as the longer-term impact of the pandemic on people with dementia is as yet unknown.

While many participants with dementia felt they coped well during the pandemic, and some indicators were better than pre-pandemic levels, there were several important perceived negative impacts that could have significant long-term consequences. The main implications of these findings are three-fold. First, the findings are consistent with perceptions of a steeper than usual trajectory of decline in cognitive and functional ability, and hence ability to manage self-care and everyday activities, resulting from the conditions experienced during periods of restriction due to the pandemic. Where there has been an accelerated decline, some lost ground might potentially be recoverable, but this would require appropriate support for reablement [54]; otherwise, more rapid and extensive decline is likely to result in increased needs for care and higher demands on family carers. Second, increased feelings of loneliness among people with dementia are a major cause for concern, especially given the established associations of loneliness with poor psychological well-being and quality of life [52] and hence the potential risks associated with experiencing loneliness. It may be that loneliness will reduce as restrictions ease and opportunities to resume social activities emerge, but here again, support may be needed to regain lost ground, especially where confidence has been lost or anxieties about contracting the virus remain high. Third, our findings demonstrate a negative impact on sense of self and on perceptions of quality of life and life satisfaction, and it is not clear whether this trend might reverse naturally; if not, additional support in the form of personalized planning might be needed to regain a better quality of life.

\section{CONCLUSIONS}

Examining the perceived impact of the restrictions imposed during the COVID-19 pandemic on people with dementia participating in the IDEAL cohort, as described through self-report and through informant reports from carers, has provided a unique opportunity to compare experiences during the pandemic with pre-pandemic data. The findings suggest that efforts to mitigate negative impacts of pandemicrelated restrictions on people with dementia living in the community could focus primarily on reablement to address the effects of accelerated declines in ability to carry out and participate in everyday activities, on creating opportunities for meaningful social contact to reduce loneliness, and on personalized planning to reconnect people with their pre-COVID selves and restore pre-COVID levels of quality of life. Such efforts may build on the resilience demonstrated by people with dementia and their carers in coping with the pandemic and, in many cases, managing to maintain a generally positive outlook on life despite the extra challenges of living with dementia under these circumstances.

\section{ACKNOWLEDGMENTS}

We acknowledge the support of NIHR Dementias and Neurodegeneration Specialty (DeNDRoN) and Health and Care Research Wales with IDEAL cohort recruitment and data collection. We gratefully acknowledge the local principal investigators and researchers involved in participant recruitment and assessment within these networks. We are grateful to the IDEAL study participants for their participation in the IDEAL and INCLUDE studies, to the wider group of IDEAL programme researchers, and to members of the ALWAYs group and the Project Advisory Group for their support. 
'Identifying and mitigating the individual and dyadic impact of COVID-19 and life under physical distancing on people with dementia and carers (INCLUDE)' was funded by the Economic and Social Research Council (ESRC) through grant ES/V00 4964/1. Investigators: Clare, L., Victor, C., Matthews, F., Quinn, C., Hillman, A., Burns, A., Allan, L., Litherland, R., Martyr, A., Collins, R., \& Pentecost, C. ESRC is part of UK Research and Innovation (UKRI).

'Improving the experience of Dementia and Enhancing Active Life: living well with dementia. The IDEAL study' was funded jointly by the Economic and Social Research Council (ESRC) and the National Institute for Health Research (NIHR) through grant ES/L001853/2. Investigators: L. Clare, I.R. Jones, C. Victor, J.V. Hindle, R.W. Jones, M. Knapp, M. Kopelman, R. Litherland, A. Martyr, F.E. Matthews, R.G. Morris, S.M. Nelis, J.A. Pickett, C. Quinn, J. Rusted, J. Thom. ESRC is part of UK Research and Innovation (UKRI). IDEAL data were deposited with the UK data archive in April 2020 and will be available to access from April 2023. Details of how the data can be accessed after that date can be found here: https://reshare.ukdataservice.ac.uk/ 854293/

'Improving the experience of Dementia and Enhancing Active Life: a longitudinal perspective on living well with dementia. The IDEAL-2 study' is funded by Alzheimer's Society, grant number 348, AS-PR2-16-001. Investigators: L. Clare, I.R. Jones, C. Victor, C. Ballard, A. Hillman, J.V. Hindle, J. Hughes, R.W. Jones, M. Knapp, R. Litherland, A. Martyr, F.E. Matthews, R.G. Morris, S.M. Nelis, C. Quinn, J. Rusted.

L. Clare acknowledges support from the NIHR Applied Research Collaboration South-West Peninsula.

The views expressed are those of the author(s) and not necessarily those of the ESRC, UKRI, NIHR, the Department of Health and Social Care, the National Health Service, or Alzheimer's Society. The support of ESRC, NIHR and Alzheimer's Society is gratefully acknowledged.

Authors' disclosures available online (https:// www.j-alz.com/manuscript-disclosures/21-5095r2).

\section{SUPPLEMENTARY MATERIAL}

The supplementary material is available in the electronic version of this article: https://dx.doi.org/ 10.3233/JAD-215095.

\section{REFERENCES}

[1] Martyr A, Nelis SM, Quinn C, Wu Y-T, Lamont RA, Henderson C, Clarke R, Hindle JV, Thom JM, Jones IR, Morris RG, Rusted JM, Victor CR, Clare L (2018) Living well with dementia: A systematic review and correlational metaanalysis of factors associated with quality of life, well-being and life satisfaction in people with dementia. Psychol Med 48, 2130-2139.

[2] Institute of Medicine (2012) Living Well with Chronic Illness: A Call for Public Health Action, National Academies Press, Washington.

[3] Clare L, Wu Y-T, Jones IR, Victor CR, Nelis SM, Martyr A, Quinn C, Litherland R, Pickett JA, Hindle JV, Jones RW, Knapp M, Kopelman MD, Morris RG, Rusted JM, Thom JM, Lamont RA, Henderson C, Rippon I, Hillman A, Matthews FE, On behalf of the IDEAL study team (2019) A comprehensive model of factors associated with subjective perceptions of "living well" with dementia: Findings from the IDEAL study. Alzheimer Dis Assoc Disord 33, 36-41.

[4] Clare L, Nelis SM, Quinn C, Martyr A, Henderson C, Hindle JV, Jones IR, Jones RW, Knapp M, Kopelman MD, Morris RG, Pickett JA, Rusted JM, Savitch NM, Thom JM, Victor CR (2014) Improving the experience of dementia and enhancing active life - living well with dementia: Study protocol for the IDEAL study. Health Qual Life Outcomes 12, 164

[5] Silarova B, Nelis SM, Ashworth RM, Ballard C, Bieńkiewicz M, Henderson C, Hillman A, Hindle JV, Hughes JC, Lamont RA, Litherland R, Jones IR, Jones RW, Knapp M, Kotting P, Martyr A, Matthews FE, Morris RG, Quinn C, Regan J, Rusted JM, van den Heuvel EA, Victor CR, Wu Y-T, Clare L (2018) Protocol for the IDEAL-2 longitudinal study: Following the experiences of people with dementia and their primary carers to understand what contributes to living well with dementia and enhances active life. BMC Public Health 18, 1214.

[6] Borges-Machado F, Barros D, Ribeiro O, Carvalho J (2020) The effects of COVID-19 home confinement in dementia care: Physical and cognitive decline, severe neuropsychiatric symptoms and increased caregiving burden. Am J Alzheimers Dis Other Demen 35, 1533317520976720.

[7] Boutoleau-Bretonniere C, Pouclet-Courtemanche H, Gillet A, Bernard A, Deruet AL, Gouraud I, Mazoue A, Lamy E, Rocher L, Kapogiannis D, El Haj M (2020) The effects of confinement on neuropsychiatric symptoms in Alzheimer's disease during the COVID-19 crisis. J Alzheimers Dis 76, 41-47.

[8] Canevelli M, Valletta M, Toccaceli Blasi M, Remoli G, Sarti G, Nuti F, Sciancalepore F, Ruberti E, Cesari M, Bruno G (2020) Facing dementia during the COVID-19 outbreak. $J$ Am Geriatr Soc 68, 1673-1676.

[9] Tsapanou A, Papatriantafyllou JD, Yiannopoulou K, Sali D, Kalligerou F, Ntanasi E, Zoi P, Margioti E, Kamtsadeli V, Hatzopoulou M, Koustimpi M, Zagka A, Papageorgiou SG, Sakka P (2021) The impact of COVID-19 pandemic on people with mild cognitive impairment/dementia and on their caregivers. Int J Geriatr Psychiatry 36, 583-587.

[10] Bacsu JD, O'Connell ME, Cammer A, Azizi M, Grewal K, Poole L, Green S, Sivananthan S, Spiteri RJ (2021) Using Twitter to understand the COVID-19 experiences of people with dementia: Infodemiology study. J Med Internet Res $\mathbf{2 3}$, e26254.

[11] Carlos AF, Poloni TE, Caridi M, Pozzolini M, Vaccaro R, Rolandi E, Cirrincione A, Pettinato L, Vitali SF, Tronconi 
L, Ceroni M, Guaita A (2021) Life during COVID-19 lockdown in Italy: The influence of cognitive state on psychosocial, behavioral and lifestyle profiles of older adults. Aging Ment Health, doi: 10.1080/13607863.2020.1870210.

[12] Roach P, Zwiers A, Cox E, Fischer K, Charlton A, Josephson CB, Patten SB, Seitz D, Ismail Z, Smith EE (2021) Understanding the impact of the COVID-19 pandemic on well-being and virtual care for people living with dementia and care partners living in the community. Dementia (London) 20, 2007-2023

[13] van Maurik IS, Bakker ED, van den Buuse S, Gillissen F, van de Beek M, Lemstra E, Mank A, van den Bosch KA, van Leeuwenstijn M, Bouwman FH, Scheltens $P$, van der Flier WM (2020) Psychosocial effects of corona measures on patients with dementia, mild cognitive impairment and subjective cognitive decline. Front Psychiatry 11, 585686.

[14] O'Rourke G, Pentecost C, Van den Heuvel EA, Victor C, Quinn C, Hillman A, Litherland R, Clare L (2021) Living with dementia under COVID-19 restrictions: Coping and support needs among people with dementia and carers from the IDEAL cohort. Ageing Soc, doi: 10.1017/S0144686X21001719.

[15] Azevedo L, Calandri IL, Slachevsky A, Graviotto HG, Vieira MCS, Andrade CB, Rossetti AP, Generoso AB, Carmona KC, Pinto LAC, Sorbara M, Pinto A, Guajardo T, Olavarria L, Thumala D, Crivelli L, Vivas L, Allegri RF, Barbosa MT, Serrano CM, Miranda-Castillo C, Caramelli P (2021) Impact of social isolation on people with dementia and their family caregivers. J Alzheimers Dis 81, 607-617.

[16] Barguilla A, Fernandez-Lebrero A, Estragues-Gazquez I, Garcia-Escobar G, Navalpotro-Gomez I, Manero RM, Puente-Periz V, Roquer J, Puig-Pijoan A (2020) Effects of COVID-19 pandemic confinement in patients with cognitive impairment. Front Neurol 11, 589901.

[17] Borelli WV, Augustin MC, de Oliveira PBF, Reggiani LC, Bandeira-de-Mello RG, Schumacher-Schuh AF, Chaves MLF, Castilhos RM (2021) Neuropsychiatric symptoms in patients with dementia associated with increased psychological distress in caregivers during the COVID-19 pandemic. J Alzheimers Dis 80, 1705-1712.

[18] Cohen G, Russo MJ, Campos JA, Allegri RF (2020) COVID-19 epidemic in argentina: Worsening of behavioral symptoms in elderly subjects with dementia living in the community. Front Psychiatry 11, 866.

[19] Hanna K, Giebel C, Tetlow H, Ward K, Shenton J, Cannon J, Komuravelli A, Gaughan A, Eley R, Rogers C, Rajagopal M, Limbert S, Callaghan S, Whittington R, Butchard S, Shaw L, Gabbay M (2021) Emotional and mental wellbeing following COVID-19 public health measures on people living with dementia and carers. J Geriatr Psychiatry Neurol, doi: $10.1177 / 0891988721996816$.

[20] Panerai S, Prestianni G, Musso S, Muratore S, Tasca D, Catania V, Gelardi D, Ferri R (2020) The impact of COVID19 confinement on the neurobehavioral manifestations of people with Major Neurocognitive Disorder and on the level of burden of their caregivers. Life Span Disabil 23, 303-320.

[21] Pongan E, Dorey JM, Borg C, Getenet JC, Bachelet R, Lourioux C, Laurent B, Group C, Rey R, Rouch I (2021) COVID-19: Association between increase of behavioral and psychological symptoms of dementia during lockdown and caregivers' poor mental health. J Alzheimers Dis 80, 17131721.

[22] Rainero I, Bruni AC, Marra C, Cagnin A, Bonanni L, Cupidi C, Lagana V, Rubino E, Vacca A, Di Lorenzo R, Provero P, Isella V, Vanacore N, Agosta F, Appollonio I,
Caffarra P, Busse C, Sambati R, Quaranta D, Guglielmi V, Logroscino G, Filippi M, Tedeschi G, Ferrarese C, Group SIC-S (2020) The impact of COVID-19 quarantine on patients with dementia and family caregivers: A nation-wide survey. Front Aging Neurosci 12, 625781.

[23] Tam MT, Dosso JA, Robillard JM (2021) The impact of a global pandemic on people living with dementia and their care partners: Analysis of 417 lived experience reports. $J$ Alzheimers Dis 80, 865-875.

[24] Thyrian JR, Kracht F, Nikelski A, Boekholt M, SchumacherSchonert F, Radke A, Michalowsky B, Vollmar HC, Hoffmann W, Rodriguez FS, Kreisel SH (2020) The situation of elderly with cognitive impairment living at home during lockdown in the Corona-pandemic in Germany. BMC Geriatrics 20, 540.

[25] Manini A, Brambilla M, Maggiore L, Pomati S, Pantoni L (2021) The impact of lockdown during SARS-CoV-2 outbreak on behavioral and psychological symptoms of dementia. Neurol Sci 42, 825-833.

[26] Giebel C, Cannon J, Hanna K, Butchard S, Eley R, Gaughan A, Komuravelli A, Shenton J, Callaghan S, Tetlow H, Limbert S, Whittington R, Rogers C, Rajagopal M, Ward K, Shaw L, Corcoran R, Bennett K, Gabbay M (2020) Impact of COVID-19 related social support service closures on people with dementia and unpaid carers: A qualitative study. Aging Mental Health 25, 1281-1288.

[27] Goodman-Casanova JM, Dura-Perez E, Guzman-Parra J, Cuesta-Vargas A, Mayoral-Cleries F, Durá-Pérez E, Guzmán-Parra J, Mayoral-Cleries F (2020) Telehealth home support during COVID-19 confinement for communitydwelling older adults with mild cognitive impairment or mild dementia: Survey study. J Med Internet Res 22 , e19434.

[28] Lara B, Carnes A, Dakterzada F, Benitez I, Piñol-Ripoll G (2020) Neuropsychiatric symptoms and quality of life in Spanish patients with Alzheimer's disease during the COVID-19 lockdown. Eur J Neurol 27, 1744-1747.

[29] Wu Y-T, Nelis SM, Quinn C, Martyr A, Jones IR, Victor CR, Knapp M, Henderson C, Hindle JV, Jones RW, Kopelman MD, Morris RG, Pickett JA, Rusted JM, Thom JM, Litherland R, Matthews FE, Clare L, on behalf of the IDEAL Programme team (2020) Factors associated with self- and informant ratings of quality of life, well-being and life satisfaction in people with mild-to-moderate dementia: Results from the Improving the experience of Dementia and Enhancing Active Life programme. Age Ageing 49, 446-452.

[30] Martyr A, Clare L (2018) Awareness of functional ability in people with early-stage dementia. Int J Geriatr Psychiatry 32, 31-38

[31] Martyr A, Nelis SM, Clare L (2014) Predictors of perceived functional ability in early-stage dementia: Self-ratings, informant ratings and discrepancy scores. Int J Geriatr Psychiatry 29, 852-862.

[32] Martyr A, Nelis SM, Quinn C, Rusted JM, Morris RG, Clare $\mathrm{L}$, on behalf of the Ideal programme team (2019) The relationship between perceived functional difficulties and the ability to live well with mild-to-moderate dementia: Findings from the IDEAL programme. Int J Geriatr Psychiatry 34, 1251-1261.

[33] Altieri M, Santangelo G (2021) The psychological impact of COVID-19 pandemic and lockdown on caregivers of people with dementia. Am J Geriatr Psychiatry 29, 27-34.

[34] Budnick A, Hering C, Eggert S, Teubner C, Suhr R, Kuhlmey A, Gellert P (2021) Informal caregivers during the 
COVID-19 pandemic perceive additional burden: Findings from an ad-hoc survey in Germany. BMC Health Serv Res 21, 353.

[35] Cagnin A, Di Lorenzo R, Marra C, Bonanni L, Cupidi C, Lagana V, Rubino E, Vacca A, Provero P, Isella V, Vanacore N, Agosta F, Appollonio I, Caffarra P, Pettenuzzo I, Sambati R, Quaranta D, Guglielmi V, Logroscino G, Filippi M, Tedeschi G, Ferrarese C, Rainero I, Bruni AC, SINdem COVID-19 Study Group (2020) Behavioral and psychological effects of coronavirus disease-19 quarantine in patients with dementia. Front Psychiatry 11, 578015.

[36] Carcavilla N, Pozo AS, Gonzalez B, Moral-Cuesta D, Roldan JJ, Erice V, Remirez AG (2021) Needs of dementia family caregivers in spain during the COVID-19 pandemic. J Alzheimers Dis 80, 533-537.

[37] Koh ZY, Law F, Chew J, Ali N, Lim WS (2020) Impact of coronavirus disease on persons with dementia and their caregivers: An audit study. Ann Geriatr Med Res 24, 316-320.

[38] Savla J, Roberto KA, Blieszner R, McCann BR, Hoyt E, Knight AL (2021) Dementia caregiving during the "stay-athome" phase of COVID-19 pandemic. J Gerontol B Psychol Sci Social Sci 76, e241-e245.

[39] Steptoe A, Addario G, Banks J, Batty GD, Coughlin K, Crawford R, Dangerfield P, Marmot M, Nazroo J, Oldfield Z, Pacchiotti B, Steel N, Wood M, Zaninotto P (2021) English Longitudinal Study of Ageing COVID-19 Study, Waves 1-2, 2020. [data collection], UK Data Service. SN: 8688.

[40] Folstein MF, Folstein SE, McHugh PR (1975) "Mini-mental state". A practical method for grading the cognitive state of patients for the clinician. J Psychiatr Res 12, 189-198.

[41] All-Party Parliamentary Group on Dementia (2013) Dementia does not discriminate: The experiences of black, Asian and minority ethnic communities, House of Commons London.

[42] Prince M, Knapp M, Guerchet M, McCrone P, Prina M, Comas-Herrera A, Wittenberg R, Adelaja B, Hu B, King D (2014) Dementia UK: Second edition - Overview, Alzheimer's Society, London.

[43] Wong A, Xiong YY, Kwan PW, Chan AY, Lam WW, Wang K, Chu WC, Nyenhuis DL, Nasreddine Z, Wong LK, Mok VC (2009) The validity, reliability and clinical utility of the Hong Kong Montreal Cognitive Assessment (HK-MoCA) in patients with cerebral small vessel disease. Dement Geriatr Cogn Disord 28, 81-87.

[44] Wong A, Black SE, Yiu SYP, Au LWC, Lau AYL, Soo YOY, Chan AYY, Leung TWH, Wong LKS, Kwok TCY, Cheung TCK, Leung KT, Lam BYK, Kwan JSK, Mok VCT (2018) Converting MMSE to MoCA and MoCA 5-minute protocol in an educationally heterogeneous sample with stroke or transient ischemic attack. Int $J$ Geriatr Psychiatry 33, 729-734.
[45] Derrick B, Russ B, Toher D, White P (2017) Test statistics for the comparison of means for two samples that include both paired and independent observations. J Modern Appl Stat Methods 16, 137-157.

[46] Derrick B, Dobson-Mckittrick A, Toher D, White P (2015) Test statistics for comparing two proportions with partially overlapping samples. J Appl Quant Methods 10, 1-14.

[47] Derrick B, White P (2018) Methods for comparing the responses from a Likert question, with paired observations and independent observations in each of two samples. Int $J$ Math Stat 19, 84-93.

[48] Tuijt R, Rait G, Frost R, Wilcock J, Manthorpe J, Walters K (2021) Remote primary care consultations for people living with dementia during the COVID-19 pandemic: Experiences of people living with dementia and their carers. $\mathrm{Br}$ J Gen Pract 71, e574-e582.

[49] Clare L, Martyr A, Morris RG, Tippett LJ (2020) Discontinuity in the subjective experience of self among people with mild-to-moderate dementia is associated with poorer psychological health: Findings from the IDEAL cohort. $J$ Alzheimers Dis 77, 127-138.

[50] Ismail II, Kamel WA, Al-Hashel JY (2021) Association of COVID-19 pandemic and rate of cognitive decline in patients with dementia and mild cognitive impairment: A cross-sectional study. Gerontology and Geriatric Medicine, doi: $10.1177 / 23337214211005223$.

[51] Tondo G, Sarasso B, Serra P, Tesser F, Comi C (2021) The impact of the COVID-19 pandemic on the cognition of people with dementia. Int J Environ Res Public Health 18, 4285 .

[52] Victor CR, Rippon I, Quinn C, Martyr A, Clare L, on behalf of the IDEAL programme team (2021) The role of subjective social status in living well for carers of people with dementia: Findings from the Improving the experience of Dementia and Enhancing Active Life programme. Int J Care Caring 5, 447-467.

[53] National Institute for Health and Care Excellence (2018) Dementia: Assessment, management and support for people living with dementia and their carers, National Institute for Health and Care Excellence, London.

[54] Clare L (2017) Rehabilitation for people living with dementia: A practical framework of positive support. PLoS Med 14, e1002245.

[55] Office for National Statistics (2010) Standard occupational classification 2010. Volume 3. The national statistics socioeconomic classification: (Rebased on the SOC2010) User Manual, Palgrave Macmillan, Basingstoke. 